\title{
A pilot study investigating early postoperative changes of plasma polyunsaturated fatty acids after laparoscopic sleeve gastrectomy
}

Mutay Aslan ${ }^{1,4^{*}}$, Ibrahim Aslan², Filiz Özcan, Ramazan Eryılmaz ${ }^{3}$, Cemal Ozben Ensari and Tuna Bilecik ${ }^{3}$

\begin{abstract}
Background: This study aimed to determine early postoperative changes of plasma polyunsaturated fatty acids (PUFAs) following laparoscopic sleeve gastrectomy (LSG).

Methods: Ten obese patients (mean BMl: $51.10 \pm 11.59 \mathrm{~kg} / \mathrm{m}^{2}$ ) underwent LSG and eleven normal weight control patients (mean BMl: $24.37 \pm 2.33 \mathrm{~kg} / \mathrm{m}^{2}$ ) underwent laparoscopic abdominal surgery. Fasting blood samples were collected prior to surgery, at day 1 after surgery and after postoperation oral feeding. Plasma levels of arachidonic acid (AA, C20:4n6), dihomo-gamma-linolenic acid (DGLA, C20:3n6), eicosapentaenoic acid (EPA, C20:5n3) and docosahexaenoic acid (DHA, C22:6n3) were determined by an optimized multiple reaction monitoring (MRM) method using ultra fast-liquid chromatography (UFLC) coupled with tandem mass spectrometry (MS/MS). Prostaglandin $E_{2}$ (PGE2) was measured in serum samples by enzyme immunoassay.
\end{abstract}

Results: A significant decrease was observed in insulin and HOMA IR levels in sleeve gastrectomy patients after postoperation oral feeding compared to preoperation. Plasma AA levels and AA/EPA ratio were significantly increased in sleeve gastrectomy patients after postoperation oral feeding compared to postoperation day 1. Serum PGE2 levels and AA/DHA ratio was significantly higher in sleeve gastrectomy patients at preoperation, postoperation day 1 and after postoperation oral feeding when compared to control group patients.

Conclusion: Increased peripheral insulin sensitivity associated with LSG may play a role in the significant increase of plasma AA levels in sleeve gastrectomy patients following postoperation oral feeding. The significant increase in PGE2 levels and AA/DHA ratio in sleeve gastrectomy group patients also confirms the presence of a proinflammatory state in obesity.

Keywords: Laparoscopic sleeve gastrectomy, Polyunsaturated fatty acids, Insulin, Prostaglandin

\section{Introduction}

The human body can produce many fatty acids except the two essential polyunsaturated fatty acids (PUFAs) which include linoleic acid (LA, C18:2n6) and alphalinolenic acid (ALA, C18:3n3) [1]. Linoleic acid is the precursor of omega-6 (n-6) series of PUFAs while ALA is the precursor of omega-3 (n-3) series of PUFAs [2]. Eicosanoids derived from n-6 PUFAs such as arachidonic acid (AA, C20:4n6) have proinflammatory and

\footnotetext{
* Correspondence: mutayaslan@akdeniz.edu.tr

'Department of Medical Biochemistry, Akdeniz University Medical Faculty, Antalya, Turkey

${ }^{4}$ Department of Biochemistry, Akdeniz University Medical School, Antalya 07070, Turkey

Full list of author information is available at the end of the article
}

immunoactive functions, whereas eicosanoids derived from n-3 PUFAs such as eicosapentaenoic acid (EPA, C20:5n3) and docosahexaenoic acid (DHA, C22:6n3) have anti-inflammatory properties, attributed to their ability to inhibit the formation of n-6 PUFA-derived eicosanoids [3]. Recent studies have documented the presence of an imbalance in PUFA levels and its correlation with visceral fat accumulation in male subjects [4]. Moreover, a correlation between acute phase proteins and serum PUFA composition was shown in morbidly obese patients [5].

Laparoscopic sleeve gastrectomy (LSG) is associated with a high rate of resolution of type 2 diabetes mellitus (T2DM) and other obesity-associated comorbidities such as hypertension and hyperlipidemia [6]. The improvement 
of insulin action occurs very early at 3-5 days following LSG with a significant reduction in insulin resistance [7]. Insulin stimulates the conversion of essential fatty acids (LA and ALA) to longer-chain PUFAs [8]. Indeed, levels of the principal n-6 PUFA, AA, are reported to be significantly lower in diabetic patients than in controls $[9,10]$. It was recently shown that insulin analog initiation therapy significantly increased plasma PUFA levels in patients with T2DM [11]. Restoration of the first phase of insulin secretion and improved insulin sensitivity in diabetic obese patients immediately after sleeve gastrectomy, before any weight loss, seem to be related to hormonal changes of possible gastric origin and is neither meal- nor weightchange-related [12]. To our knowledge no study has evaluated the effect of LSG on plasma levels of PUFAs. This study aimed to assess early postoperative effects of LSG on plasma n-6 and n-3 PUFA levels.

\section{Materials and methods Patients Study groups}

The control group included 11 patients who were admitted to Antalya Research and Education Hospital, Surgery Clinic. Patients in the control group underwent laparoscopic abdominal surgery for appendectomy $(n=5)$, cholecystectomy $(\mathrm{n}=4)$, partial cystectomy $(\mathrm{n}=1)$ and inguinal hernia repair $(\mathrm{n}=1)$. Subjects with apparent history of stroke, coronary heart disease, arrhythmia, peripheral artery disease, severe kidney dysfunction, liver disease, thyroid dysfunction, infectious disease were excluded. The body mass index (BMI) of all patients in the control group was $<30 \mathrm{~kg} / \mathrm{m}^{2}$ and all were non-smokers. Fasting blood samples were obtained from all patients at preoperation, postoperation day 1 and after postoperation oral feeding.

The sleeve gastrectomy group included 10 obese patients who were admitted to Antalya Research and Education Hospital, Endocrinology Clinic. The BMI of all patients in the sleeve gastrectomy group was $\geq 40 \mathrm{~kg} / \mathrm{m}^{2}$. All patients went through a clinical, biochemical and pre-anesthetic evaluation and subjects with apparent history of stroke, coronary heart disease, arrhythmia, peripheral artery disease, severe kidney dysfunction, liver disease, thyroid dysfunction, infectious disease were excluded. All patients met the surgical indication criteria in the inter-disciplinary European guidelines on surgery of severe obesity [13]. Fasting blood samples were obtained from all sleeve gastrectomy patients the day before operation (preoperation), the day after operation (postoperation day 1 ) and the day after postoperation oral feeding. All sleeve gastrectomy patients were on preoperative diet for 2 weeks, before surgery. This diet contained liquid protein supplements and sugar-free, noncarbonated, low calorie fluids and required a minimum of 2 liters of fluid intake daily. Female and male patients were aimed to receive 65 and 80 grams protein daily, respectively.
Patients did not receive any food and no fluid by mouth starting from midnight the day of surgery until the day after surgery. Patients were tested several times after surgery for anastomatic leaks. When patients were determined to be leak free, postoperation oral feeding was initiated by aiming $120 \mathrm{ml}$ per hour fluid intake. Clear bouillon, sugar-free gelatin, sugar-free flavored beverages, 1:1 water diluted apple, cranberry, or grape juice were allowed to be added to the diet at this stage. Postoperation oral feeding was continued until the patients were discharged from the hospital. All sleeve gastrectomy patients and control group subjects gave written informed consent prior to entry. This study was approved by the Institutional Review Board for Human Use at Akdeniz University Faculty of Medicine.

\section{Laboratory measurements}

Serum glucose was measured on Roche Cobas 8000 Modular Analyser (Basel, Switzerland). Insulin levels were measured by Roche/Hitachi E170 modular analyser (Tokyo, Japan). Insulin sensitivity was evaluated using homeostatic model assessment for insulin resistance (HOMA IR) [14].

\section{Electrospray ionization mass spectrometry}

Standards for AA (C20:4n6), DGLA (C20:3n6), EPA $(\mathrm{C} 20: 5 \mathrm{n} 3)$ and DHA (C22:6n3) were purchased from Sigma-Aldrich (St. Louis MO, USA). Deuterium labeled AA-d8 internal standard $(5,6,8,9,11,12,14,15$-AA-d8) was obtained from Santa Cruz Biotechnology (Santa Cruz, CA, USA). Solutions of AA, DGLA, EPA, DHA and AA-d8 standards were prepared in analytical grade methanol (Merck, Darmstadt, Germany). An optimized multiple reaction monitoring (MRM) method was developed using ultra-fast liquid chromatography (UFLC) coupled with tandem mass spectrometry (MS/MS). A UFLC system (LC-20 AD UFLC XR, Shimadzu Corporation, Japan) was coupled to a LCMS-8040 triple quadrupole mass spectrometer (Shimadzu Corporation, Japan). Chromatographic separations were carried out using Inertsil HPLC column (ODS-4, $2.1 \times 100 \mathrm{~mm}, 3 \mu \mathrm{m}$; GL Sciences Inc. Tokyo, Japan) maintained at $40^{\circ} \mathrm{C}$. DHA, EPA, AA and DGLA were separated using a gradient elution with a flow rate of $0.45 \mathrm{ml} / \mathrm{min}$. Mobile phase solvent A was $10 \mathrm{mM}$ ammonium acetate (Sigma-Aldrich, St. Louis, MO, USA) in water and solvent B was acetonitrile (Sigma-Aldrich, St. Louis, MO, USA). Gradient program was solvent B, 70\% (0 $\mathrm{min}$ ), $90 \%$ (3 min), 100\% (3.01-4 $\mathrm{min}$ ) and 70\% (4.01$8 \mathrm{~min})$. MRM transitions and responses were automatically optimized for individual compounds in negative electrospray ionization (ESI). In the negative ESI-MS mode the precursor and product $\mathrm{m} / \mathrm{z}$ values for AA, DHA, EPA, DGLA and AA-d8 are given in Table 1. Responses to AA, DHA, EPA and DGLA were optimized to a linear calibration range from $100 \mathrm{ng} / \mathrm{ml}$ to $30 \mathrm{ug} / \mathrm{ml}$ and a sample analysis time of 8 minutes. 
Table 1 The precursor and product $\mathrm{m} / \mathrm{z}$ values for analyzed polyunsaturated fatty acids

\begin{tabular}{lcc}
\hline & Precursor $\mathbf{~} / \mathbf{z}$ & Product $\mathbf{~} / \mathbf{z}$ \\
\hline DGLA (C20:3n6) & 304.80 & $59.00,260.70$ \\
AA (C20:4n6) & 303.10 & $59.00,258.90$ \\
EPA (C20:5n3) & 301.10 & $59.10,256.70$ \\
DHA (C22:6n3) & 327.10 & $59.10,283.20$ \\
AA-d8 & 311.10 & $59.10,97.90,267.10$
\end{tabular}

DGLA, dihomo-gamma-linolenic acid; AA, arachidonic acid; EPA, eicosapentaenoic acid; DHA, docosahexaenoic acid.

\section{Sample preparation for LC-MS/MS}

Samples were prepared for LC-MS/MS analysis via a modified protocol as previously described [15]. Briefly, in a glass test tube, $200 \mu \mathrm{l}$ plasma was added to $200 \mu \mathrm{l}$ AA-d8 internal standard solution. $1 \mathrm{ml}$ of acetonitril/ 37\% hydrochloric acid (Cayman, Ann Arbor, MI, USA) was added to the mixture in a 4:1 $\mathrm{v} / \mathrm{v}$. Tubes were capped with reusable teflon liner screw caps and samples were hydrolyzed by incubating at $90^{\circ} \mathrm{C}$ for 2 hours in a heating block (VLM, Bielefeld, Germany). After cooling down to room temperature, fatty acids were extracted with $2 \mathrm{ml}$ of hexane. Samples were vortex-mixed for 20 seconds, left at room temperature for 5 minutes and centrifuged at $3000 \mathrm{rpm}$ for 1 minute. The upper phase containing free fatty acids were transferred to glass tubes and evaporated at room temperature under a constant stream of nitrogen with height adjustable gas distribution unit (VLM, Bielefeld, Germany). Fatty acids were dissolved in $200 \mu \mathrm{l}$ methanol-water (180:20, v/v) filtered via $0,2 \mu \mathrm{m}$ polytetrafluoroethylene (PTFE) syringe filters (Whatman, GE Healthcare Bio-Sciences, Pittsburgh, USA) and transferred to autosampler vials (Vertical Chromatography, Nonthaburi, Thailand).

\section{Measurement of prostaglandin $\mathrm{E}_{2}$}

Prostaglandin $\mathrm{E}_{2}$ (PGE2) was measured in serum samples by a commercial enzyme immunoassay test kit [KGE004B; R\&D Systems, Inc., Minneapolis, MN 55413, USA] according to manufacturer's instructions. A standard curve of absorbance values of known PGE2 standards was plotted as a function of the logarithm of PGE2 standard concentrations (pg/ml) using the GraphPad Prism Software program for windows version 5,03. (GraphPad Software Inc). PGE2 concentrations in the samples were calculated from their corresponding absorbance values via the standard curve.

\section{Statistical analysis}

Data were analyzed using Sigma Stat (version 2.03) statistical software for Windows, and a $\mathrm{P}$ value $<0.05$ was considered statistically significant.

\section{Results}

Control and sleeve gastrectomy group characteristics

The control group was composed mainly of women (7 female, 4 male). The mean \pm SD of age, body weight and body mass index in the control group was $41 \pm 18$ years, $65 \pm 6 \mathrm{~kg}$ and $24.37 \pm 2.33 \mathrm{~kg} / \mathrm{m}^{2}$, respectively. The sleeve gastrectomy group was also composed mainly of women (7 female, 3 male). The mean \pm SD of age, body weight, body mass index, fat mass and lean mass in the sleeve gastrectomy group was $38 \pm 11$ years, $130 \pm 22 \mathrm{~kg}, 51.10 \pm$ $11.59 \mathrm{~kg} / \mathrm{m}^{2}, 67.2 \pm 15 \mathrm{~kg}$ and $59.7 \pm 10.3 \mathrm{~kg}$ respectively.

\section{Biochemical measurements}

Glucose, insulin and HOMA IR levels from control and sleeve gastrectomy patients are shown in Table 2. A significant decrease was observed in insulin and HOMA IR levels in sleeve gastrectomy patients after postoperation oral feeding compared to preoperation. Statistical analysis was done by Paired t-test.

\section{ESI-MS spectra}

Figure 1A shows representative negative ion mode spectra of a patient sample. As shown in the figure, retention time of EPA (C20:5n3), DHA (C22:6n3), AA (C20:4n6) and DGLA (C20:3n6) was 1.869, 2.131, 2.391 and 2.911 minutes, respectively. Figure 1B shows tandem mass spectra obtained by collision-induced dissociation of precursor ions. The $\mathrm{m} / \mathrm{z}$ values of $256.7,258.9,260.7,283.2$ product ions correspond to endogenous C20:5n3, C20:4n6, C20:3n6 and $\mathrm{C} 22: 6 \mathrm{n} 3$, respectively. The deuterium-labeled internal standard fatty acid peaks are indicated at $\mathrm{m} / \mathrm{z}$ values 97.9 and 267.1.

\section{Levels of polyunsaturated fatty acids}

Blood samples obtained from control group patients showed no significant difference in levels of AA (C20:4n6), DGLA (C20:3n6), EPA (C20:5n3) and DHA (C22:6n3) (Table 3). The ratio of DGLA/AA, AA/EPA and AA/DHA were also similar in control group samples obtained at preoperation, postoperation day 1 and after postoperation oral feeding. Likewise, DGLA (C20:3n6), EPA $(\mathrm{C} 20: 5 \mathrm{n} 3)$ and DHA (C22:6n3) serum levels in sleeve

Table 2 Serum glucose, insulin concentration and HOMA-IR values in control and sleeve gastrectomy group

\begin{tabular}{|c|c|c|c|c|}
\hline \multicolumn{3}{|c|}{ Control group } & \multicolumn{2}{|c|}{ Sleeve gastrectomy group } \\
\hline Variable & $\begin{array}{l}\text { Preop } \\
(n=11)\end{array}$ & $\begin{array}{l}\text { Po OF } \\
(n=11)\end{array}$ & $\begin{array}{l}\text { Preop } \\
(n=10)\end{array}$ & $\begin{array}{l}\text { Po OF } \\
(n=10)\end{array}$ \\
\hline Glucose (mg/dL) & $103.0 \pm 21.6$ & $96.9 \pm 22.7$ & $102.2 \pm 26.8$ & $102.5 \pm 31.3$ \\
\hline Insulin (mU/L) & $9.7 \pm 9.4$ & $7.7 \pm 6.9$ & $18.8 \pm 10.2$ & $11.1 \pm 6.9^{*}$ \\
\hline HOMA-IR & $2.7 \pm 3.3$ & $2.1 \pm 2.9$ & $4.9 \pm 3.5$ & $2.9 \pm 2.1^{*}$ \\
\hline
\end{tabular}

Values are mean \pm SD. Preop, preoperation; Postop, postoperation; Po OF, postoperation oral feeding. ${ }^{*} \mathrm{p}<0.01$ postoperation oral feeding vs. preoperation in the sleeve gastrectomy group. 


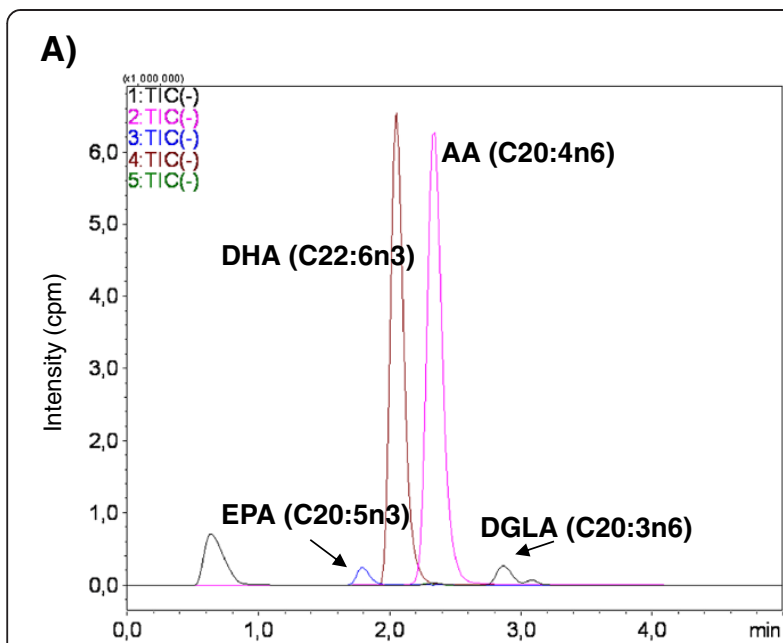

B)

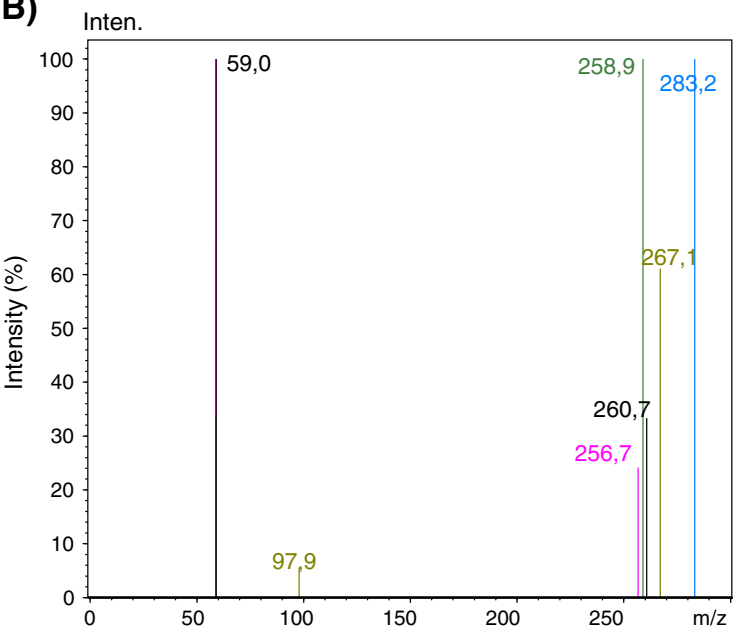

Figure 1 ESI-MS Spectra. A) Representative negative ion mode spectra of a patient sample. DGLA, Dihomo-gamma-linolenic acid; AA Arachidonic acid; EPA, Eicosapentaenoic acid; DHA, Docosahexaenoic acid. B) Tandem mass spectra. gastrectomy patients were similar at preoperation, postoperation day 1 and after postoperation oral feeding. There was also no significant difference observed in DGLA (C20:3n6), EPA (C20:5n3) and DHA (C22:6n3) serum levels between control and sleeve gastrectomy patients (Table 3). Serum AA (C20:4n6) levels and AA/EPA ratio were significantly increased in sleeve gastrectomy patients after postoperation oral feeding compared to postoperation day 1. Levels of AA (C20:4n6) and AA/EPA ratio were also significantly higher in sleeve gastrectomy patients after postoperation oral feeding compared to control group patients on the same day. AA/DHA ratio was significantly higher in sleeve gastrectomy patients at preoperation, postoperation day 1 and after postoperation oral feeding when compared to control group patients (Table 3). Statistical analysis was done by Paired t-test or Wilcoxon Signed Rank Test. Distribution of AA (C20:4n6), DGLA (C20:3n6), EPA (C20:5n3) and DHA (C22:6n3) in serum samples of control and sleeve gastrectomy patients are shown in Figure 2.

\section{Prostaglandin $\mathrm{E}_{2}$ levels}

Bar graph data of serum PGE2 content are shown in Figure 3. Serum PGE2 levels were similar at preoperation, postoperation day 1 and after postoperation oral feeding in the control group. Likewise, no significant difference was observed in serum PGE2 levels within sleeve gastrectomy patients. Serum PGE2 levels were significantly higher in sleeve gastrectomy patients at preoperation, postoperation day 1 and after postoperation oral feeding when compared to control group patients (Figure 3). Statistical analysis was done by paired t-test.

\section{Discussion}

Polyunsaturated fatty acids regulate inflammatory responses through the production of eicosanoids including

Table 3 Analysis of polyunsaturated fatty acids in control and sleeve gastrectomy group from preoperation up to postoperation oral feeding

\begin{tabular}{|c|c|c|c|c|c|c|}
\hline \multicolumn{4}{|c|}{ Control group } & \multicolumn{3}{|c|}{ Sleeve gastrectomy group } \\
\hline Variable & Preop $(n=11)$ & Postop day $1(n=11)$ & Po OF $(n=11)$ & Preop $(n=10)$ & Postop day $1(n=10)$ & Po OF $(n=10)$ \\
\hline AA (C20:4n6) $(\mu \mathrm{g} / \mathrm{ml})$ & $124.0 \pm 27.8$ & $110.5 \pm 22.8$ & $117.5 \pm 22.7$ & $143.1 \pm 44.5$ & $114.7 \pm 30.0$ & $159.7 \pm 25.0^{* a}$ \\
\hline DGLA (C20:3n6) $(\mu \mathrm{g} / \mathrm{ml})$ & $45.9 \pm 16.4$ & $37.9 \pm 13.1$ & $36.4 \pm 13.8$ & $67.4 \pm 27.2$ & $53.9 \pm 21.0$ & $47.1 \pm 16.3$ \\
\hline EPA $(C 20: 5 \mathrm{n} 3)(\mu \mathrm{g} / \mathrm{ml})$ & $9.1 \pm 7.7$ & $7.9 \pm 9.4$ & $7.2 \pm 6.4$ & $5.6 \pm 2.5$ & $4.0 \pm 1.2$ & $4.2 \pm 1.4$ \\
\hline DHA (C22:6n3) $(\mu \mathrm{g} / \mathrm{ml})$ & $50.4 \pm 13.6$ & $47.4 \pm 13.8$ & $51.1 \pm 11.2$ & $38.3 \pm 14.8$ & $33.8 \pm 11.8$ & $44.6 \pm 10.6$ \\
\hline DGLA/AA & $0.38 \pm 0.14$ & $0.34 \pm 0.11$ & $0.31 \pm 0.11$ & $0.47 \pm 0.13$ & $0.46 \pm 0.12$ & $0.31 \pm 0.15^{* *}$ \\
\hline AA/EPA & $23.7 \pm 18.1$ & $23.3 \pm 12.9$ & $24.7 \pm 12.6$ & $28.2 \pm 11.9$ & $29.2 \pm 5.6$ & $42.0 \pm 16.1 *$ \\
\hline AA/DHA & $2.64 \pm 0.94$ & $2.45 \pm 0.65$ & $2.39 \pm 0.63$ & $3.87 \pm 0.85^{\mathrm{a}}$ & $3.54 \pm 0.66^{\mathrm{a}}$ & $3.84 \pm 1.50^{\mathrm{a}}$ \\
\hline
\end{tabular}

Values are mean \pm SD. Preop, preoperation; Postop, postoperation; Po OF, postoperation oral feeding; DGLA, Dihomo-gamma-linolenic acid; AA, Arachidonic acid; EPA, Eicosapentaenoic acid; DHA, Docosahexaenoic acid.

${ }^{*} p=0.007$ postoperation oral feeding vs. postoperation day 1 in the sleeve gastrectomy group.

${ }^{* *} \mathrm{p}<0.02$ postoperation oral feeding vs. preoperation and postoperation day 1 in the sleeve gastrectomy group.

${ }^{a} p<0.02$ sleeve gastrectomy group vs. control (the same day). 


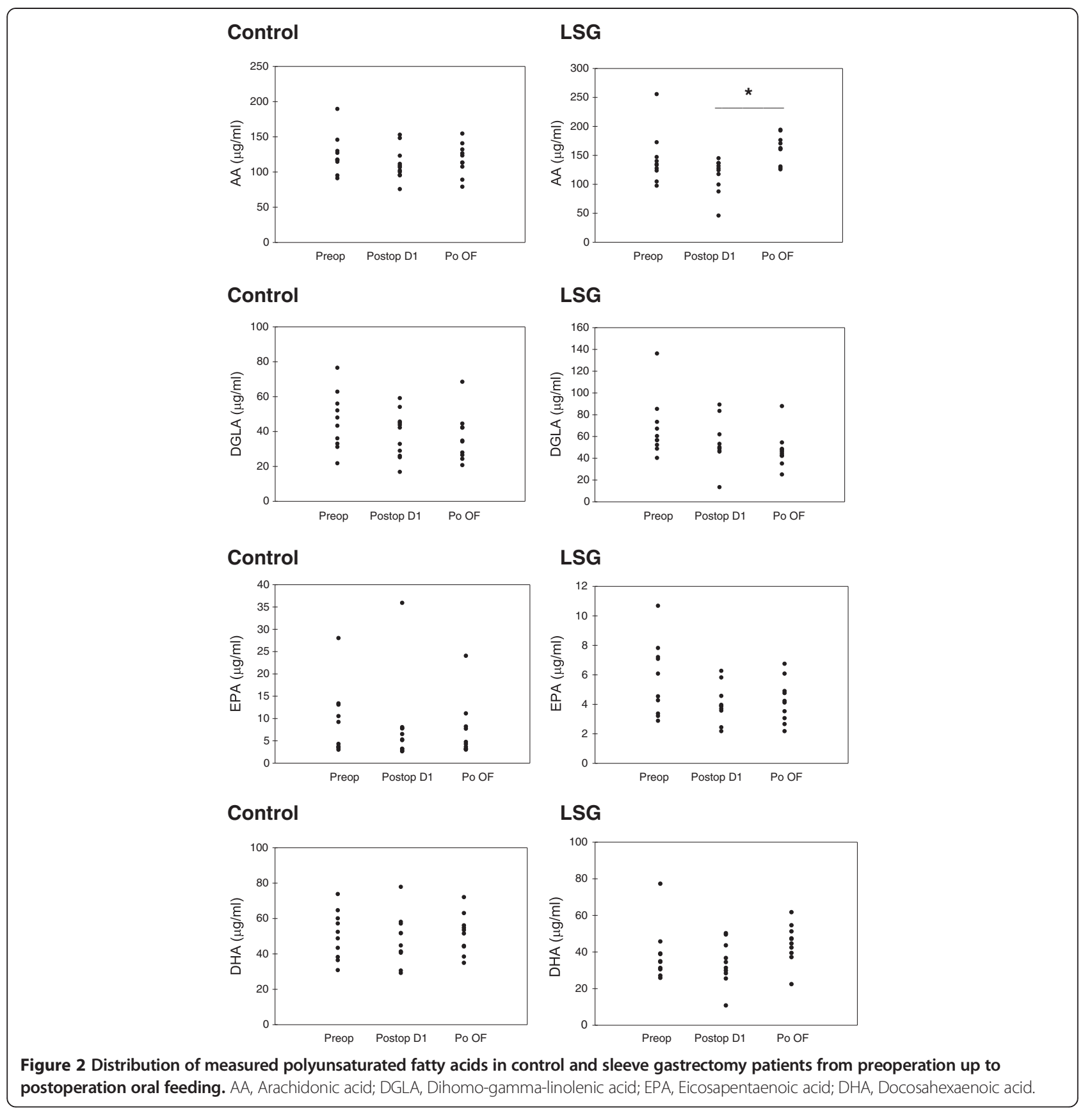

prostaglandins (PGs), thromboxanes (TXs) and leukotrienes (LTs) [2]. To our knowledge, this is the first study evaluating early postoperative effects of LSG on plasma PUFA levels. Plasma AA (C20:4n6) levels and AA/EPA ratio were significantly increased in sleeve gastrectomy patients after postoperation oral feeding compared to postoperation day 1 . The observed significant increase of plasma AA levels following LSG may be due to increased peripheral insulin sensitivity. In agreement with previous studies $[7,16]$ we have observed a significant reduction in insulin levels occurring very early at $4-5$ days following sleeve gastrectomy with a significant reduction in insulin resistance. It was recently shown that insulin initiation therapy significantly increased plasma levels of AA (C20:4n6) compared to before treatment levels in T2DM patients [11]. Disturbed fatty acid metabolism is an important feature of the insulin-resistant state [17]. Essential fatty acids are metabolized into more physiologically active compounds by introduction of further double bonds by delta-5- and delta-6-desaturase enzymes [9]. Emerging evidence shows that delta-5 desaturase is the key regulator in the synthesis of PUFA and 


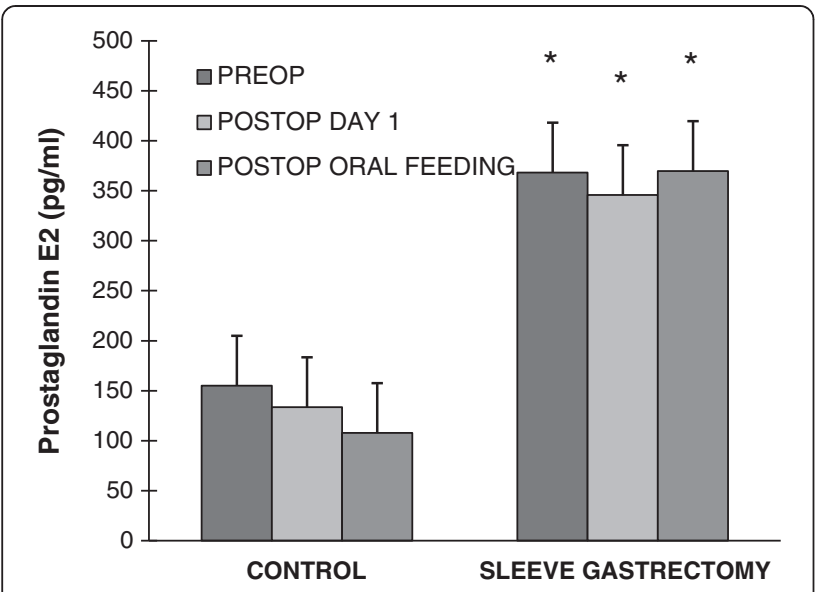

Figure 3 Prostaglandin $E_{2}$ in control and sleeve gastrectomy group from preoperation up to postoperation oral feeding ${ }^{*} p<0.05$ sleeve gastrectomy group vs. control (the same day).

is modulated by factors including adiposity, diet and insulin resistance [18]. The hepatic microsomal delta-6desaturation of LA and ALA was found to be depressed in alloxan induced diabetic rats [19]. The observed enzymatic defect was corrected by insulin injection in 2 days [20].

High levels of saturated fatty acids and low amounts of PUFAs are associated with obesity [21]. Omega-3 PUFA enriched diet increases expression of genes involved in glucose transport [glucose transporter type 4 (GLUT-4)] and insulin signaling [insulin receptor substrate 1 (IRS-1)], as well as genes involved in insulin sensitivity [peroxisome proliferator-activated receptor gamma (PPARy)] [22]. In an insulin-resistant state, omega-3 PUFAs bind to the G-protein coupled receptor 120 (GPR120), resulting in reduced cytokine production from inflammatory macrophages and improved signaling in adipocytes, leading to a reduction in insulin resistance [23]. In this study, we have observed a significant increase in AA/EPA ratio in sleeve gastrectomy patients after postoperation oral feeding compared to preoperation levels. This finding suggests that improved insulin sensitivity in obese patients immediately after sleeve gastrectomy in not related to changes in omega-3 PUFA levels.

Low serum EPA/AA ratio was recently reported in male subjects with visceral obesity [4]. Likewise, an imbalance of dietary long-chain PUFAs, especially high omega-6/ omega-3 PUFA ratio, was associated with increased risk of cardiovascular disease [24]. We have observed that AA (C20:4n6)/DHA(C22:6n3) ratio was significantly higher in sleeve gastrectomy patients at preoperation, postoperation day 1 and after postoperation oral feeding when compared to control group subjects. The significant increase of PGE2 levels and AA/DHA ratio in all performed measurements in the sleeve gastrectomy group patients supports the presence of a proinflammatory state in obesity. Competition between omega- 6 and omega- 3 fatty acids occurs in the production of eicosanoids by stereospecific lipid-oxidizing enzymes cylooxygenase (COX) and lipoxygenase (LOX) [25]. Docosahexaenoic acid (DHA, C22:6n3) is a precursor of eicosanoids with less marked inflammatory effect. On the other hand, AA is a precursor of eicosanoids with definite inflammatory effect [26]. Hence, increased AA to DHA ratio indicates more precursor for the synthesis of highly inflammatory eicosanoids.

As stated in the introduction of the manuscript, restoration of the first phase of insulin secretion and improved insulin sensitivity in diabetic obese patients immediately after sleeve gastrectomy, before any weight loss, seem to be related to hormonal changes of possible gastric origin and is neither meal- nor weight-changerelated [12]. Significantly increased AA levels in sleeve gastrectomy patients after postoperation oral feeding may play a constructive role in the observed improved insulin sensitivity in sleeve gastrectomy patients. It was previously observed that patients with type 2 diabetes mellitus have low AA content in their plasma phospholipid fraction and it was suggested that AA deficiency may predispose humans to develop T2DM [27]. In support of these findings it was recently demonstrated that insulin analog initiation therapy in T2DM patients increased AA in human plasma (11). The role of AA as a possible endogenous anti-diabetic molecule is discussed comprehensively in a recent review [28].

Limitation of our study include: 1) the study covers a small cohort; 2) the study has a nonrandomized design because patients were assigned to either group according to clinical criteria; 3) The study population included an unequal percentage of male patients. However, the limitation of gender distribution has been minimized by matching the control group similarly.

In summary, we have observed a significant decrease in insulin and HOMA IR levels in sleeve gastrectomy patients after postoperation oral feeding compared to preoperation. This was accompanied by significantly increased plasma AA levels. The significant increase in PGE2 levels and AA/DHA ratio in sleeve gastrectomy group patients also confirms the presence of a proinflammatory state in obesity.

\section{Abbreviations}

AA: Arachidonic acid; ALA: Alpha-linolenic acid; COX: Cylooxygenase; DHA: Docosahexaenoic acid; EPA: Eicosapentaenoic acid; ESI: Electrospray ionization; GLUT-4: Glucose transporter type 4; GPR120: G-protein coupled receptor 120; IRS-1: Insulin receptor substrate 1; LA: Linoleic acid;

LOX: Lipoxygenase; LSG: Laparoscopic sleeve gastrectomy; LTs: Leukotrienes; omega-3: n-3; omega-6: n-6; PGE2: Prostaglandin E2; PGs: Prostaglandins; PPARY: Peroxisome proliferator-activated receptor gamma;

PTFE: Polytetrafluoroethylene; PUFAs: Polyunsaturated fatty acids; T2DM: Type 2 diabetes mellitus; TXs: Thromboxanes. 


\section{Competing interests}

All authors declare that they have no financial, consulting, and personal relationships with other people or organizations that could influence the presented work

\section{Authors' contributions}

MA, carried out LC-MS/MS analysis, measurement of PGE2 and drafted the manuscript. FO, carried out LC-MS/MS analysis and measurement of PGE2. IA carried out the clinical studies including enrollment of patients, collection of blood samples and contributed in the drafting of the manuscript. RE, carried out the clinical studies including enrollment of patients, collection of blood samples. COE, carried out the clinical studies including enrollment of patients, collection of blood samples. TB, carried out the clinical studies including enrollment of patients, collection of blood samples. All authors read and approved the final manuscript.

\section{Acknowledgement}

This study was supported by a grant (No: 2010.04.0103.014 and 2012.06.0103.054) from Akdeniz University Research Foundation.

\section{Author details}

${ }^{1}$ Department of Medical Biochemistry, Akdeniz University Medical Faculty, Antalya, Turkey. ${ }^{2}$ Endocrinology Clinic, Antalya Research and Education Hospital, Antalya, Turkey. ${ }^{3}$ Surgery Clinic, Antalya Research and Education Hospital, Antalya, Turkey. ${ }^{4}$ Department of Biochemistry, Akdeniz University Medical School, Antalya 07070, Turkey.

Received: 6 February 2014 Accepted: 27 March 2014

Published: 3 April 2014

\section{References}

1. Patterson E, Wall R, Fitzgerald GF, Ross RP, Stanton C: Health implications of high dietary omega-6 polyunsaturated fatty acids. J Nutr Metab 2012, 2012:539426.

2. Wall R, Ross RP, Fitzgerald GF, Stanton C: Fatty acids from fish: the antiinflammatory potential of long-chain omega-3 fatty acids. Nutr Rev 2010, 68:280-289.

3. Davidson $\mathrm{MH}$ : Omega-3 fatty acids: new insights into the pharmacology and biology of docosahexaenoic acid, docosapentaenoic acid, and eicosapentaenoic acid. Curr Opin Lipidol 2013, 24:467-474.

4. Inoue K, Kishida K, Hirata A, Funahashi T, Shimomura I: Low serum eicosapentaenoic acid/arachidonic acid ratio in male subjects with visceral obesity. Nutr Metab (Lond) 2013, 10:25.

5. Fernandes R, Beserra BT, Cunha RS, Hillesheim E, Camargo Cde Q, Pequito DC, de Castro IC, Fernandes LC, Nunes EA, Trindade EB: Relationship between acute phase proteins and serum fatty acid composition in morbidly obese patients. Dis Markers 2013, 35:105-112.

6. Silecchia G, Boru C, Pecchia A, Rizzello M, Casella G, Leonetti F, Basso N: Effectiveness of laparoscopic sleeve gastrectomy (first stage of biliopancreatic diversion with duodenal switch) on co-morbidities in super-obese high-risk patients. Obes Surg 2006, 16:1138-1144.

7. Rizzello M, Abbatini F, Casella G, Alessandri G, Fantini A, Leonetti F, Basso N: Early postoperative insulin-resistance changes after sleeve gastrectomy. Obes Surg 2010, 20:50-55.

8. Brenner RR: Hormonal modulation of delta6 and delta5 desaturases: case of diabetes. Prostaglandins Leukot Essent Fatty Acids 2003, 68:151-162.

9. Jones DB, Carter RD, Haitas B, Mann Jl: Low phospholipid arachidonic acid values in diabetic platelets. Br Med J (Clin Res Ed) 1983, 286:173-175.

10. Decsi T, Minda H, Hermann R, Kozári A, Erhardt E, Burus I, Molnár S, Soltész G: Polyunsaturated fatty acids in plasma and erythrocyte membrane lipids of diabetic children. Prostaglandins Leukot Essent Fatty Acids 2002, 67:203-210.

11. Aslan M, Ozcan F, Aslan B, Yücel G: LC-MS/MS analysis of plasma polyunsaturated fatty acids in type 2 diabetic patients after insulin analog initiation therapy. Lipids Health Dis 2013, 12:169.

12. Basso N, Capoccia D, Rizzello M, Abbatini F, Mariani P, Maglio C, Coccia F, Borgonuovo G, De Luca ML, Asprino R, Alessandri G, Casella G, Leonetti F: First-phase insulin secretion, insulin sensitivity, ghrelin, GLP-1, and PYY changes $72 \mathrm{~h}$ after sleeve gastrectomy in obese diabetic patients: the gastric hypothesis. Surg Endosc 2011, 25:3540-3550.

13. Fried M, Hainer V, Basdevant A, Buchwald H, Deitel M, Finer N, Greve JW, Horber F, Mathus-Vliegen E, Scopinaro N, Steffen R, Tsigos C, Weiner R,
Widhalm K: Inter-disciplinary European guidelines on surgery of severe obesity. Int J Obes (Lond) 2007, 31:569-577.

14. Wallace TM, Levy JC, Matthews DR: Use and abuse of HOMA modeling. Diabetes Care 2004, 27:1487-1495.

15. Valianpour F, Selhorst JJ, van Lint LE, van Gennip AH, Wanders RJ, Kemp S: Analysis of very long-chain fatty acids using electrospray ionization mass spectrometry. Mol Genet Metab 2003, 79:189-196.

16. Doğan S, Aslan I, Eryılmaz R, Ensari CO, Bilecik T, Aslan M: Early postoperative changes of HDL subfraction profile and HDL-associated enzymes after laparoscopic sleeve gastrectomy. Obes Surg 2013, 23:1973-1980.

17. Rimoldi OJ, Finarelli GS, Brenner RR: Effects of diabetes and insulin on hepatic delta6 desaturase gene expression. Biochem Biophys Res Commun 2001, 283:323-326.

18. Zhou YE, Kubow S, Dewailly E, Julien P, Egeland GM: Decreased activity of desaturase 5 in association with obesity and insulin resistance aggravates declining long-chain n-3 fatty acid status in Cree undergoing dietary transition. Br J Nutr 2009, 102:888-894.

19. Poisson JP: Comparative in vivo and in vitro study of the influence of experimental diabetes on rat liver linoleic acid delta 6- and delta 5desaturation. Enzyme 1985, 34:1-14

20. Mercuri O, Peluffo RO, Brenner RR: Depression of microsomal desaturation of linoleic to gamma-linolenic acid in the alloxan-diabetic rat. Biochim Biophys Acta 1966, 116:409-411.

21. Micallef M, Munro I, Phang M, Garg M: Plasma n-3 polyunsaturated fatty acids are negatively associated with obesity. Br J Nutr 2009, 102:1370-1374.

22. Kazemian P, Kazemi-Bajestani SM, Alherbish A, Steed J, Oudit GY: The use of $\omega$-3 poly-unsaturated fatty acids in heart failure: a preferential role in patients with diabetes. Cardiovasc Drugs Ther 2012, 26:311-320.

23. González-Périz A, Horrillo R, Ferré N, Gronert K, Dong B, Morán-Salvador E, Titos E, Martínez-Clemente M, López-Parra M, Arroyo V, Clària J: Obesity induced insulin resistance and hepatic steatosis are alleviated by omega-3 fatty acids: a role for resolvins and protectins. FASEB J 2009, 23:1946-1957.

24. Ueeda M, Doumei T, Takaya Y, Ohnishi N, Takaishi A, Hirohata S, Miyoshi T, Shinohata R, Usui S, Kusachi S: Association of serum levels of arachidonic acid and eicosapentaenoic acid with prevalence of major adverse cardiac events after acute myocardial infarction. Heart Vessels 2011, 26:145-152

25. Calder PC: Polyunsaturated fatty acids, inflammation, and immunity Lipids 2001, 36:1007-1024.

26. Bagga D, Wang L, Farias-Eisner R, Glaspy JA, Reddy ST: Differential effects of prostaglandin derived from omega- 6 and omega- 3 polyunsaturated fatty acids on COX-2 expression and IL-6 secretion. Proc Natl Acad Sci U S A 2003, 100:1751-1756.

27. Das UN: Essential fatty acid metabolism in patients with essential hypertension, diabetes mellitus and coronary heart disease. Prostaglandins Leukot Essent Fatty Acids 1995, 52:387-391.

28. Das UN: Arachidonic acid and lipoxin A4 as possible endogenous antidiabetic molecules. Prostaglandins Leukot Essent Fatty Acids 2013, 88:201-210.

\section{doi:10.1186/1476-511X-13-62}

Cite this article as: Aslan et al:: A pilot study investigating early postoperative changes of plasma polyunsaturated fatty acids after laparoscopic sleeve gastrectomy. Lipids in Health and Disease 2014 13:62.

\section{Submit your next manuscript to BioMed Central and take full advantage of:}

- Convenient online submission

- Thorough peer review

- No space constraints or color figure charges

- Immediate publication on acceptance

- Inclusion in PubMed, CAS, Scopus and Google Scholar

- Research which is freely available for redistribution 